\title{
The Bioinformatics Analysis in the Whole Genome mtDNA Sequence of Chickens (Gallus gallus)
}

\author{
Suhardi Suhardi ${ }^{1 *}$, Widya Pintaka Bayu Putra ${ }^{2}$, Rofik Rofik ${ }^{3}$, Servis Simanjuntak ${ }^{1}$, \\ Khoiru Indana ${ }^{1}$, and Ari Wibowo ${ }^{1}$
}

\author{
${ }^{1}$ Department of Animal Science, Agriculture Faculty, Mulawarman University, Samarinda, Indonesia, 75123 \\ ${ }^{2}$ Research Center for Biotechnology, Indonesian Institute of Science, Bogor, Indonesia, 16911 \\ ${ }^{3}$ Livestock and Animal Health Services, Provincial Government of East Kalimantan, Bhayangkara Rd. No.54, \\ Samarinda, East Kalimantan, Indonesia 75121 \\ *Corresponding author. Email: suhardi@faperta.unmul.ac.id
}

\begin{abstract}
Chicken is important livestock in the world that is kept for egg and meat productions. This study was aimed to perform a bioinformatics analysis in the whole genome mtDNA (16,979 bp) of many chicken breeds. Total 84 whole-genome mtDNA sequences were used in this study and obtained from the reference sequence (GenBank). Four molecular packages of BioEdit, MEGA-X, DNAsp, and Arlequin were used in this study to analyze the observed sequences. Therefore, the whole genome mtDNA sequence of birds in this study was originated from Asia, the USA, and New Guinea. Research showed that a total of 445 mutation sites and 81 haplotypes were obtained in this study. Thus, the nucleotide diversity in the observed sequences included of high (0.99). In addition, a total of 10 clusters were observed according to the phylogenetic analysis with the UPGMA method. In conclusion, a close genetic relationship was observed among chickens of Asia based on the median-joining network. Meanwhile, the geographical factors were contributed about $9.12 \%$ in a sequence variation.
\end{abstract}

Keywords: Bioinformatics, Chicken, GenBank, Genetic diversity, mtDNA.

\section{INTRODUCTION}

The physical geography of an area affects the number of species of inhabitants [1]. During the last decade conservation organizations and government agencies have worked to identify, prioritize, manage and restore wild species and habitats. Many countries have begun to commit to emphasizing research on biodiversity mapping [2]. Biodiversity decline is of concern for several reasons. Most immediately, many people depend directly on biodiversity for food, fibre, fuel, and medicines [3]. Chicken is one of the most popular biodiversity varieties in the field of animal husbandry [4].

Chicken is a type of poultry that breeds or varieties that are spread almost all over the world. The chickens were domesticated from a common ancestor, the Red Jungle Fowl (Gallus gallus), which originates from Southeast Asia [5, 6]. Moreover, with the massive use of selection and crossbreeding, local breeds and lines in different parts of Europe were developed, and Asian breeds of the Chinese and Malay types were introduced [7]. All of these sources contributed to the modern biodiversity of chicken populations. Inter-crossing, however, may have partly extinguished differences among groups or breeds, with the result that genetic relationships between chicken populations are not always definitive $[8,9,10]$.

In this world, there are four types of jungle fowl, namely: gray jungle fowl (Gallus sonneratii Temminck), orange jungle fowl (Gallus lafayetii Lesson), chicken red jungle fowl (Gallus gallus Linnaeus), and green jungle fowl (Gallus varius Shaw and Nodder) [11]. The four types of jungle fowl are classified into: genus Gallus, family Phasianidae, and Order Galliformes [12]. The poultry industry comprises two major elements, the egg industry, and the chicken meat industry.

The science of bioinformatics can be divided into several branches based on the experimental material used for the study [13]. Bioinformatics is an interdisciplinary field that develops methods and software tools for understanding biological data. As an interdisciplinary field of science, bioinformatics combines computer science, statistics, mathematics, and engineering to 
analyze and interpret biological data [14]. Bioinformaticians need a solid background in computer science but also a good understanding of biology. Since bioinformaticians work closely with biologists, they need the ability to communicate complex topics in an understandable way and keep up-to-date with new developments in biology [15].

Based on the explanation above, the aim of the research is to perform a bioinformatics analysis in the whole genome mtDNA (16,979 bp) of many chicken breeds. In addition, bioinformatics assignment analysis was also performed to evaluate the genetic structure of the whole genome mtDNA sequence of chickens (Gallus gallus).

\section{MATERIALS AND METHODS}

Total 84 whole genome of mtDNA sequence of chickens $(16,797 \mathrm{bp})$ from Asia, New Guinea and USA were used in this study and obtained from the reference sequence (GenBank) as listed in the Table 1. Therefore, the bioinformatic analysis was performed using four molecular packages of BioEdit [16], MEGA-X [17], DNAsp [18] and Arlequine [19]. Thus, these packages were used to obtain the phylogenetic tree, polymorphism statistical analysis, median joining network and analysis of molecular (AMOVA).

\section{RESULT AND DISCUSSION}

This study was intended to observe the mate-pairs both having positions in the same chromosome were scanned against the reference genome by analyzing the deviations in insert size across the genome of chicken. Arrangements of chicken orthologous protein-coding genes illustrate the anticipated design of grouping conservation, with the most elevated character in proteincoding exons and negligible identity in introns [20]. A Certainty interim of 2.5 standard deviations absent from the normal embed estimate at both the tails was utilized to control the scattering of the information after calling the insertion and deletion. The certainty interim was calculated for each line independently. The haplotype of some chicken breeds based on the whole genome mtDNA sequence can be seen in Table 1 .

The certainty interim was too utilized to form clusters of insertions or deletions utilizing various leveled clustering, where the deviations of mate-pair remove from the normal embed estimate was utilized to develop a similarity index. This approach might recognize tall number of cancellations as compared to additions. Considering combined rate of recognized indels in High growth line (HL), the low growth line (LL). Research showed that a total of 445 mutation sites and 81 haplotypes were obtained in the 84 whole genome of mtDNA sequence of chickens (Figure 1). The chicken reference genome has a substantial number of incorrectly duplicated sequences, according to analysis of the single read data previously generated (artificial duplications) [20]. This study were formed analysis molecular variance (AMOVA) in the whole genome of mtDNA in chicken from India (18 sequences), Southeast Asia/New Guinea (13 sequences), USA (5 sequences) and China/Japan (43 sequences). Overlaps between this study recognized indels and these counterfeit duplications, considering that the counterfeit duplications secured as it were $105 \mathrm{Mbp}$, an cover of $30 \%$ of indels is to a much higher division of covers than would be anticipated by chance. In this way, it is likely that the mistakes within the chicken genome gathering, such as the manufactured duplications bestow an vital source of false-positive indels recognized within the current investigation.

The majority of deletions (96\%) were discovered in the $5 \mathrm{kbp}$ to $12 \mathrm{kbp}$ size range, while the majority of insertions $(95 \%)$ were located in the $1.8 \mathrm{kbp}$ to $3.3 \mathrm{kbp}$ size range. In comparison to $\mathrm{HL}$, large insertions of greater than $4 \mathrm{~kb}$ were more common in LL. Most pairedreads $(96 \%)$ deviating from typical insert size that supported evidence of insertion ranged from 9 to 310 for insertions, whereas most deletions $(98 \%)$ were supported by paired-reads ranging from ten to 300 in $\mathrm{HL}$ and LL, with 88 percent of deletions seen in the range from 12 to 298 (Figure 1). The standard deviation is determined by the size of the insertion/deletion and the number of paired-reads that differ from the normal insert size. The majority of standard deviations for insertions and deletions were in the range of six to twenty for insertions and 7 to 47 for deletions.

The deletions and insertions were used to identify the deletions and insertions, although the coverage of reads from the matching pipeline on each base pair position in the deletion area did not support all of the regions to be deleted, as many sequence reads were mapped within the allegedly deleted region [21]. In HL, a sub-analysis was carried out on chromosome 15. On chromosome 14, which has 358 areas diagnosed as deletions using matepair reads, the read coverage from the matching pipeline was determined. For chromosome 15 in the HL, the areas overlapping with the duplicated regions in the chicken genome were deleted in a sub-analysis. In putative deleted areas found using paired reads, a median coverage of zero (of reads mapped by the matching pipeline) resulted in 95 deleted regions.

The chicken genome contains 1.1 gigabytes [22]. The chromosome 1 is the biggest, at $200 \mathrm{Mbp}$. The pipeline takes a long time to conduct the analysis, and this chromosome uses a lot of memory. In order to finish the study of larger chromosomes with smaller chromosomes, memory and time resources for scanning the entire chicken genome for insertions and deletions must be optimized. When compared to the methodology of calling deletions based on the coverage of reads over sections of the genome, our method found a substantial number of deletions. In domestic hens, only 1300 
deletions bigger than 100 base pairs were discovered [23]. 140,484 polymorphisms in unique DNA were discovered after analyzing small insertions and deletions [24].
It is conceivable that most of the cancellations are untrue positives and assists refinement within the approach is required to extend the likelihood of finding especially genuine erasures and insertions. One of the

Table 1. The haplotype of some chicken breeds based on the whole genome mtDNA sequence1

\begin{tabular}{|c|c|c|c|c|c|c|c|c|c|}
\hline No. & Breed / Species & Origin & GenBank & Hap. & No. & Breed / Species & Origin & GenBank & Hap. \\
\hline 1 & Gallus spadiceus & - & AP003321 & 1 & 43 & Jabouillei & China & GU261696 & 43 \\
\hline 2 & Gallus gallus & - & AP003322 & 2 & 44 & Hainan & China & KY039436 & 44 \\
\hline 3 & Murghi & India & GU261709 & 3 & 45 & Gallus spadiceus & China & GU261690 & 45 \\
\hline 4 & Murghi & India & GU261707 & 4 & 46 & Gallus spadiceus & China & GU261693 & 46 \\
\hline 5 & Haringhata Black & India & KP211420 & 5 & 47 & Gallus spadiceus & China & GU261692 & 47 \\
\hline 6 & Kadaknath & India & KP211425 & 6 & 48 & Tibetian & China & MK163562 & 48 \\
\hline 7 & Ghagus & India & KP211419 & 7 & 59 & Huaiyang & China & GU261701 & 49 \\
\hline 8 & Murghi & India & GU261708 & 8 & 50 & Hengshan & China & KP244335 & 50 \\
\hline 9 & Gallus gallus & India & KP211423 & 9 & 51 & Hunan & China & GU261681 & 51 \\
\hline 10 & Aseel & India & KP211418 & 10 & 52 & Huang Lang & China & KF954727 & 52 \\
\hline 11 & Nicobari Brown & India & KP211422 & 11 & 53 & Jiangbian & China & GU261714 & 53 \\
\hline 12 & Gallus gallus & India & GU261685 & 12 & 54 & Jiangbian & China & GU261713 & 54 \\
\hline 13 & Gallus gallus & India & GU261698 & 13 & 55 & Jinhu Wufeng & China & KR347464 & 55 \\
\hline 14 & Gallus gallus & India & HQ857212 & 14 & 56 & Nandan & China & KP269069 & 56 \\
\hline 15 & Gallus gallus & India & HQ857209 & 15 & 57 & Shouguang & China & MK163560 & 57 \\
\hline 16 & Gallus galus & India & HQ857210 & 16 & 58 & Taoyuan & China & KF981434 & 58 \\
\hline 17 & Gallus gallus & India & HQ857211 & 17 & 59 & Tibetian & China & DQ648776 & 59 \\
\hline 18 & Gallus gallus & India & GU261680 & 18 & 60 & Xuefeng & China & KF826490 & 36 \\
\hline 19 & Gallus gallus & India & GU261697 & 19 & 61 & Xuefeng & China & GU261675 & 60 \\
\hline 20 & Tellicherry & India & KP211424 & 20 & 62 & Wuding & China & GU261676 & 61 \\
\hline 21 & Gallus gallus & Philippine & KY039399 & 21 & 63 & Xianju & China & GU261677 & 62 \\
\hline 22 & Gallus gallus & Indonesia & KY039425 & 22 & 64 & Yunnan & China & GU261702 & 63 \\
\hline 23 & Gallus gallus & Vietnam & KY039430 & 23 & 65 & Gallus spadiceus & China & GU261706 & 64 \\
\hline 24 & Gallus gallus & Laos & AP003319 & 24 & 66 & Gushi & China & GU261678 & 65 \\
\hline 25 & Gallus gallus & Laos & GU261687 & 25 & 67 & Yunnan & China & GU261702 & 66 \\
\hline 25 & Gallus gallus & Laos & GU261682 & 26 & 68 & Tengchongxue & China & GU261689 & 67 \\
\hline 27 & Gallus gallus & Thailand & MG605671 & 27 & 69 & Tengchongxue & China & GU261688 & 68 \\
\hline 28 & Gallus gallus & Myanmar & GU261700 & 28 & 70 & Chigulu & China & GU261705 & 69 \\
\hline 29 & Gallus gallus & Myanmar & GU261691 & 29 & 71 & Chigulu & China & GU261684 & 70 \\
\hline 30 & Gallus gallus & Myanmar & GU261704 & 30 & 72 & Chigulu & China & GU261719 & 71 \\
\hline 31 & Gallus spadiceus & Myanmar & GU261716 & 31 & 73 & Chigulu & China & GU261715 & 72 \\
\hline 32 & Gallus spadiceus & Myanmar & GU261703 & 32 & 74 & Chigulu & China & GU261717 & 73 \\
\hline 33 & Silky & Japan & AB086102 & 33 & 75 & Wenshanshandi & China & GU261699 & 74 \\
\hline 34 & Gallus gallus & $\begin{array}{l}\text { New } \\
\text { Guinea }\end{array}$ & KY039394 & 34 & 76 & Nixi & China & GU261711 & 75 \\
\hline
\end{tabular}


conceivable perplexing variables within the data set of indels recognized utilizing mapping distances between mate pairs may well be the holes and copied locales within the chicken genome, as such blunders are not considered within the calculation of huge indel scanning. exclude those with coverage in the suspected deletion region, the regions with a median coverage of zero were chosen, and 73 on chromosome 13 in HL were determined to be among them. Further research can be done to determine the precise breakpoints of the

Table 2. Analysis molecular variance (AMOVA) in the whole genome of mtDNA in chicken from India (18 sequences), Southeast Asia/New Guinea (13 sequences), USA (5 sequences) and China/Japan (43 sequences)

\begin{tabular}{|l|c|c|c|c|}
\hline \multicolumn{1}{|c|}{ Source of Variation } & $\begin{array}{c}\text { Degree of } \\
\text { Freedomm }\end{array}$ & $\begin{array}{c}\text { Sum of } \\
\text { Squares }\end{array}$ & $\begin{array}{c}\text { Variance } \\
\text { Components }\end{array}$ & $\begin{array}{c}\text { Percentage of } \\
\text { Variation (\%) }\end{array}$ \\
\hline Among populations & 3 & 140.74 & 1.74 & 9.12 \\
\hline Within populations & 78 & 1354.86 & 17.37 & 90.88 \\
\hline Total & 81 & 1495.60 & 19.11 & 100.00 \\
\hline
\end{tabular}

The pairing pipeline discards the majority of the reads because one of the pairings does not align in the genome. This could influence insertion and deletion scans because most of the reads of typical insert size that are lost will reduce coverage in the putative indel region, causing the heterozygous indel to be mistaken for a fixed putative indel [25]. Indel sizes can potentially be miscalculated as a result of this. Because some people may be segregating for the non-inserted/deleted allele, using a pooled sample can lead to the identification of some indels as heterozygous. Because the sequencing was done on a pooled sample, it's probable that some heterozygous indels were mistaken for homozygous indels. The indel's zygosity is also influenced by the confidence interval of the entire mate-pair library and the significance threshold.

This will necessitate more research to refine the settings, as well as some extra processes to identify real homozygous indels. In addition to the studies performed with the big indel tool, mate-pairs from the pairing pipeline were used to check the coverage of reads from the matching pipeline in the regions indicated as probable deletions by the mtDNA lite large indel tool. Some of them received a lot of attention. As a result, if some of them are found to be false positive deletions, more investigation is required to rule them out. Because some mate-pairs match incorrectly in the pairing pipeline and are considered significantly deviated from the normal insert size by the large-indel tool, one of the possible reasons for detecting a high number of false positives in regions along duplications in the genome is because some of the mate-pairs match incorrectly in the pairing pipeline and are considered significantly deviated from the normal insert size by the large-indel tool. Analysis of short insertions and deletions revealed the presence of high numbers of tandem duplications.

There is also some extra quality-based filtering of the reads that is required [26]. Further analysis was carried out to delete duplicated areas in chromosome 13, which resulted in the elimination of 83 deletions while reads from the matching pipeline were still present in the putative deletions discovered by the large-indel tool. To deletions. We propose aligning the unmapped reads in the genome by separating them into two portions and allowing a space between them that is likely present in the genome assembly due to pertinent indels. In the artificial genome, there are over 8000 indels of chicken genome [27]. Furthermore, the high standard deviation of the insert size in the three chicken lines also limit the possibility of finding true indels.

The reference genome assembly mapping, the algorithm used for matching the reads to the reference genome, and the algorithm and parameters used to scan for the genome for identifying probable insertions and deletions all play a role in indel detection utilizing matepairs. DNAsp Lite's matching algorithm and large-indeltool take into account the chicken genome's gaps and duplications while searching for insertions and deletions. In the chicken genome, artificial duplications have been discovered as an artifact [28]. It's probable that the gap and duplication regions will make true indel detection more difficult. Splitting the readings into equal sections of $23 \mathrm{bp}$ each and realigning them with the reference genome to determine the indels' breakpoints is one method for identifying actual indels [29].

Due to not all reads are created equal, it's possible that only a small percentage of them will be identified. Because all reads are not uniformly separated after splitting from one other to cover the deletion or insertion, this may only identify a small number of reads. The resequencing for each line provided about $7 x$ coverage, which is insufficient to align a significant number of reads to detect breakpoints using the split sequence technique. Following the discovery of breakpoints, multiple new fake reference sequences might be generated, each corresponding to the sequence surrounding a putative indel breakpoint discovered using the splitseq method. These artificial references might then be used to map any unmapped reads that have a read pair that was mapped in the matching pipeline using an alignment approach that allows gaps in the alignments to be inserted. This will make it possible to calculate the percentage of reads in the pool that have been deleted. The evidence is backed up by the reads. 
This will permit measuring the rate of peruses within the pool having erasure. The peruses supporting the prove of an erasure contribute to the factual centrality of the finding so this approach will moreover make it conceivable to identify homozygous cancellations within the lines. Inclusions and cancellations detailed in this think about may have an expansive number of untrue positives and elective calculations and approaches (considering the scope from coordinating pipeline and split-sequence examination) may be required to discover genuine critical putative insertions and deletions while reads from the matching pipeline were still present in the putative deletions discovered by the large-indel tool. To exclude those with coverage in the suspected deletion region, the regions with a median coverage of zero were chosen, and 77 on chromosome 15 in HL were determined to be among them. Further research can be done to determine the precise breakpoints of the deletions.

Overall, we observe a significant underrepresentation of genes that are extensively conserved and were likely present in chicken the mammal-bird common ancestor among the predicted chicken genes. the geographical factors were contributed about $9.12 \%$ in a

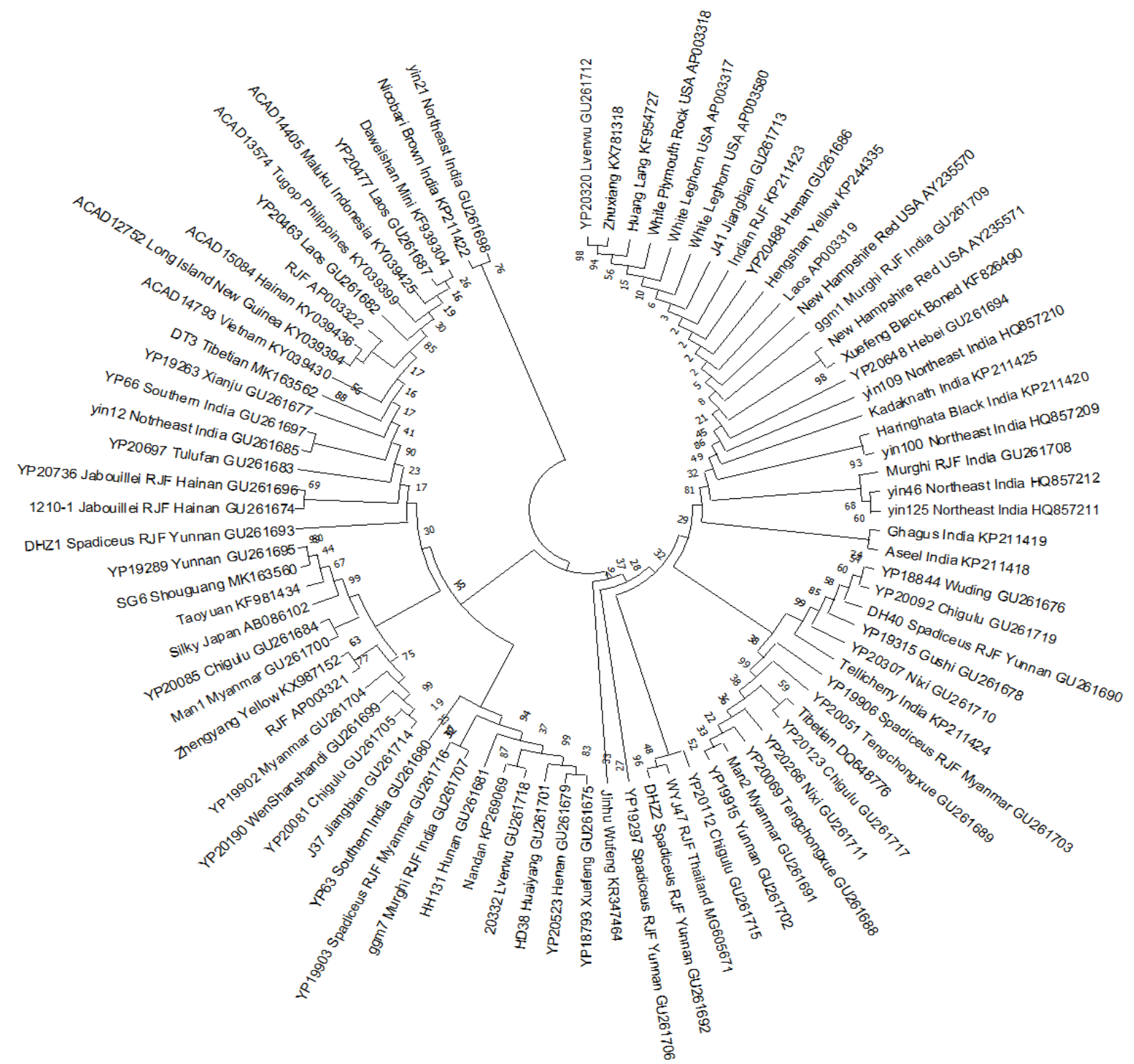

Figure 1. The circular dendogram (UPGMA 1000 bootstrap replications) of some chicken breeds based on the whole genome mtDNA

There is also some further read filtering that is required based on quality values [30]. Further analysis was carried out to delete duplicated areas in chromosome 15 , which resulted in the elimination of 85 deletions sequence variation. The mtDNA maternal lineages are thought to have originated in the Indian subcontinent [31]. As a result, it's possible that certain chickens in India, Southeast Asia/New Guinea, China/Japan, and the 
American are descended from the most common haplotype in chickens found throughout the world, which may have its origins on the Indian subcontinent.

In the mtDNA of the chicken populations, there was very little polymorphism. The chicken breeds of northeast India and Nicoobari brown India are distantly linked to red jungle fowl. All of the Asia chickens were grouped together in one region, suggesting that they came from the Asia subcontinent. Our findings will be useful in determining the mtDNA haplotypes of Asian, American, and New Guinea chickens, as well as in researching the origins of domestic chickens.

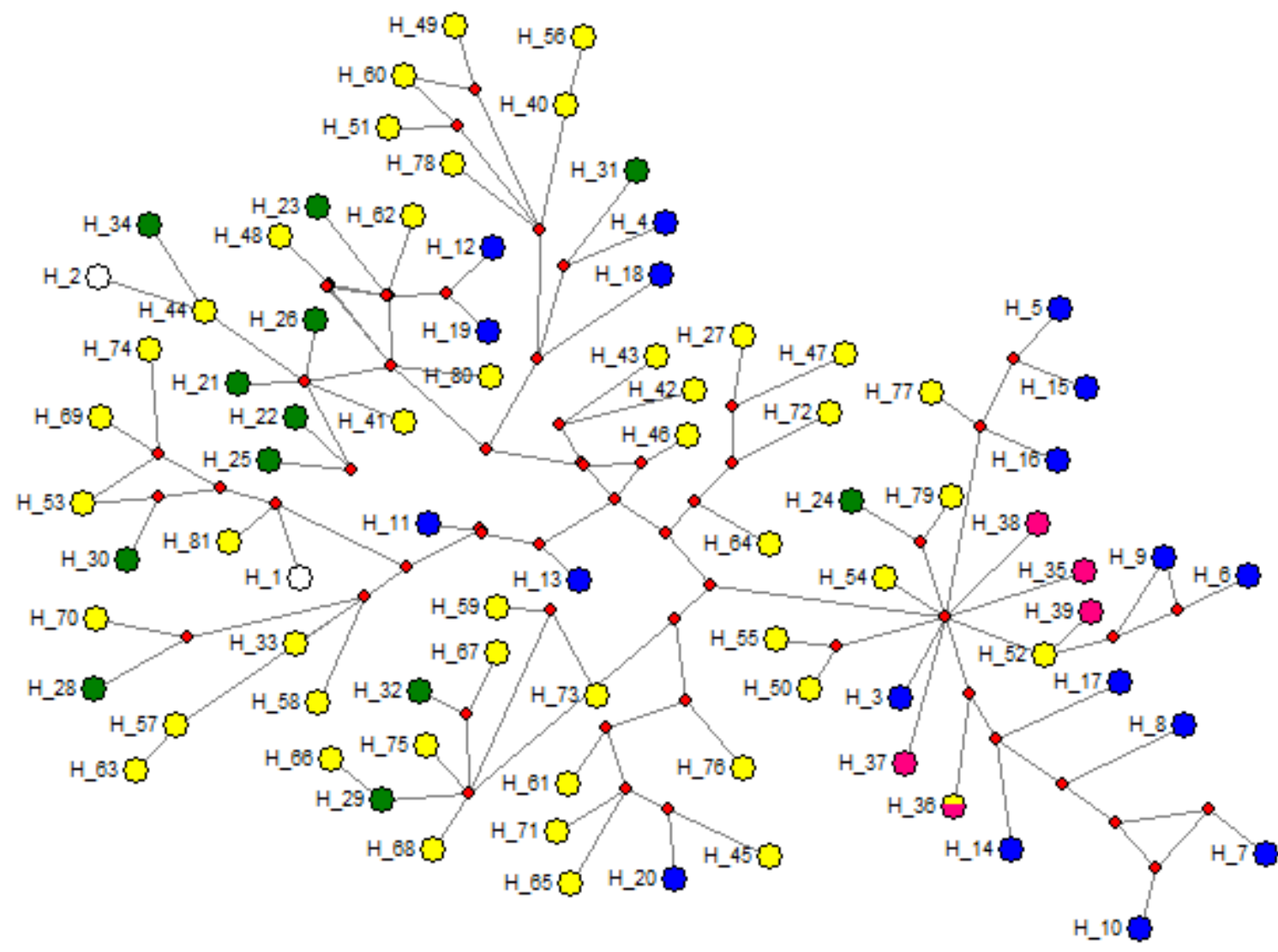

Figure 2. Median joining network of chicken breeds from some regions of India (blue), Southeast Asia/New Guinea (green), China/Japan (yellow) and USA (pink). White circle is other population

\section{CONCLUSION}

The results of this study were shown that the close genetic relationship was observed among chickens of Asia based on the median-joining network. Meanwhile, the geographical factors were contributed about $9.12 \%$ in a sequence variation.

\section{AUTHORS' CONTRIBUTIONS}

Suhardi conceptualization of the study, acquisition, analysis and interpretation of data. Widya Pintaka Bayu Putra formulation of proposed strategies, manuscript writing, as well as finalization of edits and revisions necessary. Rofik provided substantial contribution to theory development and organization of data. Servis Simanjuntak and Khoiru Indana provided material discussion on theory development and critical evaluation of research data and content. Ari Wibowo critical evaluation of research data and content, and facility sourcing as well as checking for manuscript revisions necessary.

\section{ACKNOWLEDGMENTS}

The authors thanked the Faculty of Agriculture, Mulawarman University. We are grateful to the Indonesia National Research and Innovation Agency (BRIN) for the assistance in literature, data gathering, and conceptualization of real-life scenarios of the bioinformatics genome mtDNA sequence of chickens. 
We also thank the anonymous referees for their helpful comments.

\section{REFERENCES}

[1] Mace Georgina M., Collen Ben, Fuller Richard A. and Boakes Elizabeth H. 2010Population and geographic range dynamics: implications for conservation planningPhil. Trans. R. Soc. B3653743-3751.

http://doi.org/10.1098/rstb.2010.0264

[2] Dueñas, M-A., D.J., Hemming, A., Roberts, and H., Diaz-Soltero. 2021. "The threat of invasive species to IUCN-listed critically endangered species: A systematic review." Global Ecology and Conservation. 26:e1476. doi: https://doi.org/10.1016/j.gecco.2021.e01476. [Crossref], [Web of Science $®]$, [Google Scholar].

[3] Naeem, S., Bunker, D., Hector, A., Loreau, M. \& Perrings, C. (ed.) 2009 Biodiversity, ecosystem functioning, and human wellbeing: an ecological and economic perspective. Oxford, UK: Oxford University Press.

[4] Hillel, J., Groenen, M. A., Tixier-Boichard, M., Korol, A. B., David, L., Kirzhner, V. M., Burke, T., Barre-Dirie, A., Crooijmans, R. P., Elo, K., Feldman, M. W., Freidlin, P. J., Mäki-Tanila, A., Oortwijn, M., Thomson, P., Vignal, A., Wimmers, K., \& Weigend, S. 2003. Biodiversity of 52 chicken populations assessed by microsatellite typing of DNA pools. Genetics, selection, evolution : GSE, 35(5), 533-557. https://doi.org/10.1186/1297-968635-6-533.

[5] Fumihito A., Miyake T., Sumi S., Takada M., Ohno S., Kondo N., One subspecies of the red jungle fowl (Gallus gallus gallus) suffices as the matriarchic ancestor of all domestic breeds, Proc. Natl. Acad. Sci. USA. 91 (1994) 12505-12509.

[6] Fumihito A., Miyake T., Takada M., Shingu R., Endo T., Gojobori T., Kondo N., Ohno S., Monophyletic origin and unique dispersal patterns of domestic fowls, Proc. Natl. Acad. Sci. USA 93 (1996) 6792-6795.

[7] Hunton P., Industrial breeding and selection, in: Crawford R.D. (Ed.), Poultry Breeding and Genetics. 1990. Elsevier, New York, pp. 1-42.

[8] Delacour J. 1977. Pheasants of the word. Second edition. England: World Pheasant Association and Sarga.

[9] Krane, D.E., Raymer, M.L. 2003, Fundamental Concepts of Bioinformatics, San Francisco: Benjamin Cummings, ISBN 0-8053-4633-3.
[10] Benton D. 1996. Bioinformatics - principles and potential of a new multidisciplinary tool. Trends Biotech. 14:261-312.

[11] E.M. Clarke, E.A. Emerson, Design and synthesis of synchronization skeletons using branching time temporal logic, in: D. Kozen (Eds.), Workshop on Logics of Programs, Lecture Notes in Computer Science, vol. 131, Springer, Berlin, Heidelberg, 1981, pp. 52-71. DOI: https://doi.org/10.1007/BFb0025774.

[12] J.P. Queille, J. Sifakis, Specification and verification of concurrent systems in CESAR, in: M. DezaniCiancaglini and U. Montanari (Eds.), Proceedings of the 5th International Symposium on Programming, Lecture Notes in Computer Science, vol. 137, Springer, Berlin, Heidelberg, 1982, pp. 337-351. DOI: https://doi.org/10.1007/3-540-11494-7_22.

[13] C. Baier, J-P. Katoen, Principles of Model Checking, MIT Press, 2008.

[14] M. Kwiatkowska, G. Norman, D. Parker, Stochastic model checking, in: M. Bernardo, J. Hillston (Eds.), Proceedings of the Formal Methods for the Design of Computer, Communication and Software Systems: Performance Evaluation (SFM), Springer, Berlin, Heidelberg, 2007, pp. 220-270. DOI: https://doi.org/10.1007/978-3-540-72522-0_6.

[15] V. Forejt, M. Kwiatkowska, G. Norman, D. Parker, Automated verification techniques for probabilistic systems, in: M. Bernardo, V. Issarny (Eds.), Proceedings of the Formal Methods for Eternal Networked Software Systems (SFM), Springer, Berlin, Heidelberg, 2011, pp. 53-113. DOI: https://doi.org/10.1007/978-3-642-21455-4_3.

[16] G.D. Penna, B. Intrigila, I. Melatti, E. Tronci, M.V. Zilli, Bounded probabilistic model checking with the muralpha verifier, in: A.J. Hu, A.K. Martin (Eds.), Proceedings of the Formal Methods in Computer-Aided Design, Springer, Berlin, Heidelberg, 2004, pp. 214-229. DOI: https://doi.org/10.1007/978-3-540-30494-4_16.

[17] E. Clarke, O. Grumberg, S. Jha, et al., Counterexample-guided abstraction refinement, in: E.A. Emerson, A.P. Sistla (Eds.), Computer Aided Verification, Springer, Berlin, Heidelberg, 2000, pp. 154-169.

DOI: https://doi.org/10.1007/10722167_15.

[18] H. Barringer, R. Kuiper, A. Pnueli, Now you may compose temporal logic specifications, in: Proceedings of the Sixteenth Annual ACM Symposium on the Theory of Computing (STOC), ACM, 1984, pp. 51-63. DOI: https://doi.org/10.1145/800057.808665. 
[19] A. Pnueli, In transition from global to modular temporal reasoning about programs, in: K.R. Apt (Ed.), Logics and Models of Concurrent Systems, Springer, Berlin, Heidelberg, 1984, pp. 123-144. DOI: https://doi.org/10.1007/978-3-642-82453$1 \_5$.

[20] B. Meyer, Applying "Design by Contract", Computer 25(10) (1992) 40-51. DOI: https://doi.org/10.1109/2.161279.

[21] S. Bensalem, M. Bogza, A. Legay, T.H. Nguyen, J. Sifakis, R. Yan, Incremental component-based construction and verification using invariants, in: Proceedings of the Conference on Formal Methods in Computer Aided Design (FMCAD), IEEE Press, Piscataway, NJ, 2010, pp. 257-256.

[22] H. Barringer, C.S. Pasareanu, D. Giannakopolou, Proof rules for automated compositional verification through learning, in Proc. of the 2nd International Workshop on Specification and Verification of Component Based Systems, 2003.

[23] M.G. Bobaru, C.S. Pasareanu, D. Giannakopoulou, Automated assume-guarantee reasoning by abstraction refinement, in: A. Gupta, S. Malik (Eds.), Proceedings of the Computer Aided Verification, Springer, Berlin, Heidelberg, 2008, pp. 135-148. DOI: https://doi.org/10.1007/978-3-54070545-1_14.

[24] Zody M. C., Meadows, J. R. S., Sherwood, E., Sharpe, T., Rubin, C.J., Eriksson, J., Lindblad-Toh, K., Leif Andersson. Evaluation of the Chicken Genome Assembly with SOLiD Resequencing Data.

[25] Hillier, L.W., et al. (2004) Sequence and comparative analysis of the chicken genome provide unique perspectives on vertebrate evolution. Nature: 432, 695-716.

[26] Rubin, C., Zody, M.C., Eriksson, J., Meadows, J.R.S., Sherwood , E., Webster, M.T., Jiang, L., Ingman, M., Sharpe, T., Ka, S., Hallböök, F., Besnier, F., Carlborg, Ö., Bed'hom, B., TixierBoichard, M., Jensen, P., Siegel, P., Lindblad-Toh K., \& Andersson L. (2010) Whole-genome resequencing reveals loci under selection during chicken domestication. Nature, 464, 587-591.

[27] Brandström, M. and Ellegren, H. (2007) The genomic landscape of short insertion and deletion polymorphisms in the chicken (Gallus gallus) genome: A High Frequency of deletions in tandem duplicates. Genetics, 176: 1691-1701.
[28] Sasson, A. and Michael, P. M. (2010) Filtering error from SOLiDTM output. Bioinformatics, 26:849850 .

[29] Ameur A, Wetterbom A, Feuk L, Gyllensten U. Global and unbiased detection of splice junctions from RNA-seq data. Genome Biol. 2010 Mar 17;11(3):R34.

[30] Sasson, A. and Michael, P. M. (2010) Filtering error from SOLiDTM output. Bioinformatics, 26:849850.

[31] Liu, Y. P., G. S. Wu, Y. G. Yao, Y. W. Miao, G. Luikart, M. Baig, A. Beja-Pereira, Z. L. Ding, M. G. Palanichamy, and Y. P. Zhang. 2006. Multiple maternal origins of chickens: out of Asian jungles. Mol. Phylogenet. Evol. 38:12-19. 